\title{
Gravitational kinks in two spacetime dimensions
}

\author{
M Vasilić † and T Vukašinac $\ddagger$ \\ Department of theoretical physics, Institute "Vinča", \\ P.O.Box 522, Belgrade, Yugoslavia
}

\begin{abstract}
The properties of gravitational kinks are studied within some simple models of two dimensional gravity. In spacetimes of cylindrical topology we prove the existence of kinks of constant curvature with arbitrary kink numbers. In $R^{1} \times R^{1}$ spacetimes $m=1$ kink solutions of the equation $R=0$ are found, whereas $|m|>1$ flat kinks are proved not to exist. We give a detailed analysis of the behaviour of gravitational kinks under coordinate transformations. Viewed as nonsingular black holes $|m|>1$ kink solutions are found within a simple dilaton gravity theory. The general form of the potential function is determined from the demand that the theory possesses an arbitrary number of inequivalent kink configurations.
\end{abstract}

PACS numbers: 0450, 0240

† E-mail: mvasilic@rt270.vin.bg.ac.yu

† E-mail: tatjanav@rt270.vin.bg.ac.yu 


\section{Introduction}

Over the years there has been a considerable interest in two dimensional gravity theories. This is primarily because, apart from being interesting in themselves, these models are simple enough to serve as useful tools for studying effects of mathematically more complicated four dimensional spacetime physics.

The two dimensional problem we are concerned with in this paper is related to the existence of four dimensional gravitational kinks of Finkelstein and Misner [1]. In a number of papers $[2-9]$ the properties of these topologically nontrivial metric configurations have been analysed. They have been shown to possess a twisting light-cone structure with regions of anomalous causality. Each twist, being surrounded by a one-way surface through which causes can propagate in only one direction, is an example of a nonsingular black hole. A dynamical model containing solutions of this type is, however, missing. It has further been shown that the configuration space of four dimensional kinks is double connected providing a basis for the existence of double-valued wave functions. Unfortunately, the hope that gravitational kinks could be quantized as fermions has disappeared after it has been realized that each homotopy class of metric configurations contains a spherically symmetric one [10]. Spherical symmetry of four dimensional kinks tells us that they are basically two dimensional objects. The mechanism for their existence is a feature of two dimensional spacetime. Indeed, it has been shown in reference [11] that two dimensional metrics with Minkowski signature possess similar homotopy structure to that of four dimensional case.

The purpose of this paper is to study the properties of two dimensional gravitational kinks within some simple physical models. In section two the homotopy structure of the space of metric components is analysed and the generalized Liouville equation in spacetimes of $R^{1} \times S^{1}$ topology is solved showing that gravitational kinks of constant curvature do exist on a cylinder. These solutions are, however, inappropriate to be viewed as nonsingular black holes since their horizons are uniformly disposed over the space. Section three is devoted to the analysis of kink-type nonsingular black holes in spacetimes of trivial topology. The equation $R=0$ is solved using generalized light cone coordinates. Only one-kink regular solutions are found to exist in accordance with the simple observation that more than one kink is needed to form a genuine black hole. The transformation properties of gravitational kinks are analysed in detail. In section four a model of two dimensional gravity coupled to a nonphysical dilaton field is considered. The potential term is determined from the requirement that the theory admits an arbitrary number of inequivalent stationary kink configurations. 


\section{Kinks on a cylinder}

In two spacetime dimensions the space of components of the metric tensor $g_{\mu \nu}$ is three dimensional. There is, however, a forbidden region in this space defined by vanishing metric determinant. The corresponding equation $g \equiv g_{00} g_{11}-\left(g_{01}\right)^{2}=0$ defines a coneshaped two dimensional surface which devides the space of metric components into three separate regions each characterized by a definite signature. The figure 1 clearly shows that the only topologically nontrivial region is that of Minkowski signature. It is obviously multiply connected with the first homotopy group being the group of integers.

In spacetimes of $R^{1} \times S^{1}$ topology the corresponding configuration space is represented by closed loops which completely lie in one connected region. A noncontractible loop, $m$ times wound around the forbidden surface $g=0$, is called a kink and is attributed the kink number $m$. Let us choose representatives, one in each homotopy class of metric configurations, to be

$$
g_{\mu \nu}^{m} \equiv\left(\begin{array}{cc}
\cos m \sigma & \sin m \sigma \\
\sin m \sigma & -\cos m \sigma
\end{array}\right)
$$

where $\sigma$ runs from 0 to $2 \pi$ and $m$ is an integer. The metric (1) is represented by a loop which leaves the point $g_{\mu \nu}=\eta_{\mu \nu}$ (denoted by $M$ in figure 1 ), winds $m$ times around the surface $g=0$ and ends in $g_{\mu \nu}=\eta_{\mu \nu}$ again. It is obvious that no continuous coordinate transfomation can map kinks with different kink numbers into each other. The discrete transformation of time reversal, however, maps kinks into antikinks [1]. The light cone structure of the $g_{\mu \nu}^{m}$ kinks is found to be defined by the equation

$$
\frac{d \tau}{d \sigma}=\frac{\cos m \sigma}{\sin m \sigma \pm 1} .
$$

As shown in figure 2 the light cones twist along the $\sigma$-axis just as light cones of Finkelstein and Misner do. The $m$-kink is characterised by $m / 2$ twists.

Let us now try to find a physical model possessing kink solutions on a cylinder. The simplest equation $R=0$ is a two dimensional analogue of the free Einstein equation in four dimensions. Can gravitational kinks on a cylinder be flat? As shown in reference [11] any two dimensional metric with Minkowski signature can be written in the form

$$
g_{\mu \nu}=e^{\varphi}\left(\begin{array}{cc}
\cos \psi & \sin \psi \\
\sin \psi & -\cos \psi
\end{array}\right) .
$$

One of the functions $\varphi$ or $\psi$ could further be specified but the usual choice $\psi=0$ is too restrictive excluding all kink metrics. Our choice of gauge,

$$
g_{\mu \nu}=e^{\varphi} g_{\mu \nu}^{m}
$$


is quite acceptable in that respect, although also incomplete. With this gauge choice the equation $R=0$ reduces to

$$
\stackrel{m}{\square} \varphi+m^{2} \cos m \sigma=0
$$

where $\stackrel{m}{\square}$ stands for the covariant D'Alambertian of the metric $g_{\mu \nu}^{m}$. To solve the equation (3) we shall make use of the generalized light cone coordinates

$$
x^{ \pm} \equiv \frac{1}{\sqrt{2}}\left[\tau-\frac{1}{m} \ln (1 \mp \sin m \sigma)\right]
$$

obtained by integrating the equations (2). The new coordinates are well defined only in regions of nonvanishing $\cos m \sigma$. Skipping the details of the calculation we just present the result. The general solution of the equation (3) is given by

$$
\varphi=-m \tau+A\left(x^{+}\right)+B\left(x^{-}\right)
$$

where $A$ and $B$ are two arbitrary functions. Since our light cone coordinates are defined only between zeros of $\cos m \sigma$ we must separately check the regularity of $\varphi$. A simple observation

$$
\bar{x}^{ \pm} \equiv e^{-m \sqrt{2} x^{ \pm}}=(1 \mp \sin m \sigma) e^{-m \tau}
$$

leads us directly to the manifestly regular form of $\varphi$,

$$
\varphi=-m \tau+\bar{A}\left(\bar{x}^{+}\right)+\bar{B}\left(\bar{x}^{-}\right) .
$$

Therefore, flat kinks do exist on a cylinder. Note, that this conclusion is not in discrepancy with the known result that it is always possible to find coordinate transformations which map an arbitrary flat metric into the Minkowski metric. Namely, this theorem is valid only locally and only in simply connected regions of a manifold. This is certainly not the case with the regions which accommodate kinks on a cylinder.

Let us briefly mention in the end of this section that kinks of constant curvature also exist on a cylinder. The equation $R=\lambda$ leads to the generalized Liouville equation

$$
\stackrel{m}{\square} \varphi-\lambda e^{\varphi}+m^{2} \cos m \sigma=0
$$

with the general solution of the form

$$
\lambda e^{\varphi}=\frac{4}{\cos m \sigma} \frac{\left(\partial_{+} A\right)\left(\partial_{-} B\right)}{(1-A B)^{2}} .
$$

A tedious analysis shows that the functions $A\left(x^{+}\right)$and $B\left(x^{-}\right)$can be chosen to ensure regularity of the solutions. 


\section{Kinks in $R^{1} \times R^{1}$ spacetimes}

Gravitational kinks on a cylinder are not good analogues of realistic nonsingular black holes because their horizons are disposed all over the space. For that reason, in this section, we analyse $2 \mathrm{~d}$ gravitational kinks in spacetimes of trivial topology. Homotopy considerations are the same as in the preceding section provided the boundary values of the metric are kept fixed. In each homotopy class we choose an asymptotically Minkowskian representative

$$
g_{\mu \nu}^{(m)} \equiv\left(\begin{array}{cc}
\cos m \theta & \sin m \theta \\
\sin m \theta & -\cos m \theta
\end{array}\right)
$$

with $\theta$ given by $\theta \equiv \pi+2 \operatorname{arctg} \sigma$ and $\sigma$ running from $-\infty$ to $+\infty$. It has been suggested [11] that one could make use of a singular coordinate transformation to obtain $R^{1} \times R^{1}$ kinks from $R^{1} \times S^{1}$ ones. We do not use this technique because it leads to geodesically incomplete solutions even in the asymptotic region which is highly undesirable if we want to view gravitational kinks as analogues of realistic black holes. Instead, we restrict ourselves to the metric configurations of the form

$$
g_{\mu \nu}=e^{\varphi} g_{\mu \nu}^{(m)}
$$

The light cone structure of this geometry has the twisting nature of figure 2 but is asymptotically Minkowskian. The lines $\cos m \theta=0$ define the horizons of the corresponding black hole.

Now, let us try to solve the equation $R=0$ in the gauge (4). First, we solve the equation $d s^{2}=0$ and define the light cone coordinates

$$
y^{ \pm} \equiv \frac{1}{\sqrt{2}}\left[\tau-s_{m}(\mp \theta)\right]
$$

with

$$
s_{m}(\theta) \equiv \int \frac{\cos m \theta d \theta}{(1-\cos \theta)(1+\sin m \theta)} .
$$

The coordinates $y^{ \pm}$are well defined only between zeros of the function $\cos m \theta$. The equation $R=0$, rewritten as

$$
\stackrel{(m)}{\square} \varphi-(\cos m \theta)^{\prime \prime}=0
$$

(primes denote differentiation over $\sigma$ ) has a particular solution of the form

$$
\varphi_{\text {par }}=-\ln |\cos m \theta|
$$


On the other hand, the homogeneous equation is most easily solved in the light-cone coordinates (5) in which

$$
\stackrel{(m)}{\square} \varphi=\frac{2}{\cos m \theta} \partial_{+} \partial_{-} \varphi .
$$

Using this fact we find the general solution of the equation (7) to be

$$
\varphi=A\left(y^{+}\right)+B\left(y^{-}\right)-\ln |\cos m \theta| .
$$

This solution is manifestly regular only in regions of nonvanishing $\cos m \theta$. Is it possible to choose the functions $A$ and $B$ and make $\varphi$ regular everywhere?

In the simplest case $m=1$ one easily solves the integral (6) and finds

$$
y^{ \pm}=\frac{1}{\sqrt{2}}(\tau \pm \sigma-2 \ln |\sigma \pm 1|)
$$

whereas the particular solution (8) boils down to

$$
\varphi_{\text {par }}=\frac{1}{\sqrt{2}}\left(y^{+}+y^{-}\right)+\ln \left(1+\sigma^{2}\right)-\tau .
$$

Consequently, the general solution (9) can be rewritten in the form

$$
\varphi=C\left(y^{+}\right)+D\left(y^{-}\right)+\ln \left(1+\sigma^{2}\right)-\tau
$$

or, if we use the coordinates

$$
\bar{y}^{ \pm} \equiv e^{-\sqrt{2} y^{ \pm}}=(1 \pm \sigma)^{2} e^{-\tau \mp \sigma}
$$

in the manifestly regular form

$$
\varphi=\bar{C}\left(\bar{y}^{+}\right)+\bar{D}\left(\bar{y}^{-}\right)+\ln \left(1+\sigma^{2}\right)-\tau .
$$

By choosing the functions $\bar{C}$ and $\bar{D}$ to be everywhere well defined we prove the existence of flat $m=1$ kinks in spacetimes of trivial topology. An example of this kind is considered in reference [11].

Although kinks cannot be deformed into no-kink configurations the fact that $R=0$ tells us that there must exist a continuous transformation which maps between (10) and the Minkowski solution. It is not difficult to explain the apparent inconsistency of this result. As we mentioned at the beginning of this section the homotopy considerations in topologically trivial spacetimes make sense only if the boundary values of the metric are kept fixed. The fact that a one-kink solution can be transformed into a no-kink solution 
only means that the corresponding coordinate transformation does change the asymptotics. To see this we shall make use of figure 2 where twisting light cones of an $m=1$ kink are displayed, and try to draw purely spacelike and timelike coordinate lines. These are necessary ingredients of any coordinate system in which light cones do not twist. The result is shown in figure 3. We see that it is indeed possible to construct no-kink coordinates but also that it is impossible to do so without moving the points of $\sigma$-infinity. The analysis of figure 3 is in agreement with Finkelstein's argument that spacetimes which admit a global family of spacelike surfaces do not have actual kinks [10].

Similar analysis can also be done for $|m|>1$. The set of timelike and spacelike lines for a two-kink metric is shown in figure 4 . We see that these lines cannot be used as coordinate lines of a global coordinate system. Consequently, it is not possible to transform $m=2$ kinks (and the same holds for $|m|>2$ ) into no-kink configurations. We conclude that regular $|m|>1$ solutions of the equation $R=0$ do not exist. To support this conclusion we shall analyse the behaviour of the general solution (9) in the neighbourhood of the light cone singular points. Without loss of generality we restrict ourselves to the case $m=2$. It is easy, then, to show that there are four singular points - horizons,

$$
\begin{array}{ll}
\sigma_{1}=-(1+\sqrt{2}) & \sigma_{2}=1-\sqrt{2} \\
\sigma_{3}=-(1-\sqrt{2}) & \sigma_{4}=1+\sqrt{2}
\end{array}
$$

which devide the $\sigma$-axis into five separate regions. In each region we are free to choose the functions $A$ and $B$ independently. To be able to analyse their behaviour in the vicinity of the horizons we first solve the integral (6) and find the explicit form of the light cone coordinates:

$$
\begin{aligned}
& y^{+} \sim \sigma_{1} \ln \left|\sigma-\sigma_{1}\right|-\sigma_{3} \ln \left|\sigma-\sigma_{3}\right| \\
& y^{-} \sim \sigma_{2} \ln \left|\sigma-\sigma_{2}\right|-\sigma_{4} \ln \left|\sigma-\sigma_{4}\right| .
\end{aligned}
$$

At the same time the behaviour of the particular solution (8) is given by

$$
\ln |\cos 2 \theta| \sim \ln \left|\sigma-\sigma_{1}\right|+\ln \left|\sigma-\sigma_{2}\right|+\ln \left|\sigma-\sigma_{3}\right|+\ln \left|\sigma-\sigma_{4}\right|
$$

We see that the functions $A$ and $B$ must diverge linearly to cancel the infinite contributions of (11). Let us consider the following two regions of the $\sigma$-axis: $\left(\sigma_{1}, \sigma_{2}\right)$ and $\left(\sigma_{2}, \sigma_{3}\right)$. The corresponding $A$ functions we shall denote by $A_{1}$ and $A_{2}$. Then, the requirement that the function $\varphi$ be finite at the points $\sigma=\sigma_{1}$ and $\sigma=\sigma_{3}$ gives

$$
A_{1} \sim \frac{1}{\sigma_{1}} y^{+} \text {and } A_{2} \sim-\frac{1}{\sigma_{3}} y^{+}
$$


We notice that the rates of decrease of the two functions differ. On the other hand, the demand for continuity of the function $\varphi$ at the point $\sigma=\sigma_{2}$, where $y^{+}$is finite, gives

$$
A_{1}\left(y^{+}\right)=A_{2}\left(y^{+}\right)+\text {const }
$$

This constraint implies identical asymptotic behaviour of the functions $A_{1}$ and $A_{2}$, which contradicts their formerly established asymptotics. We conclude that regular two-kink solutions of the equation $R=0$ in topologically trivial spacetimes do not exist. The same holds for $|m|>2$.

For the analysis we just presented the gauge choice (4) was not essential. There are metric configurations, not covered by our gauge choice, which also have twisting light cones even if their kink number is zero. An example is the loop of figure $1 n$ times wound around the forbidden surface and then $n$ times unwound in the opposite direction. Such configurations do not admit global families of spacelike lines and, consequently, cannot be flat. They are examples of $m=0$ nonsingular black holes.

\section{Kinks in dilatonic gravity}

In quest of a physical model accommodating gravitational kinks as analogues of four dimensional nonsingular black holes we shall consider a simple dilaton gravity theory given by the action

$$
I=\int d^{2} x \sqrt{-g}[\phi R+V(\phi)]
$$

This kind of theory has already been considered in reference [12] where the form of the potential has been determined from the requirement that the theory admits nonsingular black hole solutions. Similar constructions of nonsingular black holes can also be found in references [13-18]. All these, however, are not kink type solutions. Following the idea of [12], recently developed in [19], we search for the potential $V(\phi)$ which admits stationary kink configurations. Variation of the action (12) yields the field equations

$$
R+\frac{d V}{d \phi}=0
$$

and

$$
\nabla_{\mu} \nabla_{\nu} \phi+\frac{1}{2} g_{\mu \nu} V(\phi)=0
$$

only two of which are independent. As in the preceding section we choose the gauge (4). Then, the most general stationary solution is obtained by substituting $\phi=\phi(\sigma)$ 
and $\varphi=\varphi(\sigma)$ into the field equations. Combining the time-time and the space-space components of the equation (14) we find

$$
\phi^{\prime \prime}-\varphi^{\prime} \phi^{\prime}=0
$$

wherefrom either $\phi=$ const. or

$$
e^{\varphi}=\alpha \phi^{\prime} \quad \alpha=\text { const }
$$

The value of the potential $V(\phi)$ can also be explicitely expressed in terms of the dilaton field $\phi$ by combining the time-time and the space-time components of (14). The result is

$$
\left(\phi^{\prime} \cos m \theta\right)^{\prime}=e^{\varphi} V(\phi)
$$

Let us first consider the simplest case $\phi=$ const. Then, the full set of field equations is equivalent to

$$
\phi=\phi_{0} \quad V\left(\phi_{0}\right)=0 \quad R=-\left(\frac{d V}{d \phi}\right)_{0} \equiv \lambda
$$

where $\phi_{0}$ and $\lambda$ are constants. If $\lambda=0$, we have already seen that kink solutions with $|m|>1$ do not exist. If $\lambda \neq 0$, the stationary equation $R=\lambda$ boils down to

$$
\left[\frac{1}{\omega}(\omega \cos m \theta)^{\prime}\right]^{\prime}=\omega
$$

where $\omega \equiv \lambda e^{\varphi}$ and, consequently, $\omega \neq 0$ everywhere. When $|m|>1$ the function $\omega \cos m \theta$ changes sign at least four times. Then, $(\omega \cos m \theta)^{\prime}$ changes sign at least three times and so does $(\omega \cos m \theta)^{\prime} / \omega$. It follows then that its derivative must change sign at least twice which is, according to (18), in contradiction with $\omega \neq 0$ everywhere. Thus, there are no $|m|>1$ kink solutions of the equations (17).

The case $\phi \neq$ const. is easily shown to be completely described by the equations (15) and (16) alone. To simplify further analysis we shall make use of the prepotential function $W(\phi)$ defined as

$$
\alpha V(\phi) \equiv \frac{d W(\phi)}{d \phi}
$$

The equation (16) is then easily integrated out to give

$$
\phi^{\prime} \cos m \theta=W(\phi)
$$

Without loss of generality we set the integration constant to zero. According to (15) the dilaton field $\phi(\sigma)$ is a monotonous function of $\sigma$ taking values in the interval $D \equiv\left(\phi_{-}, \phi_{+}\right)$ 
defined by the endpoints $\phi(-\infty)$ and $\phi(+\infty)$. If the interval $D$ is finite we see that $W(\phi)$ must tend to zero as $\phi$ approaches its boundary. If $D$ is infinite the function $W(\phi)$ can go to infinity but not faster than $\mathcal{O}(\phi)$. It follows also that the prepotential function $W(\phi)$ must have the same number of zeros on $D$ as $\cos m \theta$ does on the whole $\sigma$-axis. Let us denote these zeros by $\phi_{i}$ and $\sigma_{i}$ respectively $(i=1, \ldots, 2 m)$. Taking the derivative of (20) then gives another constraint on $W(\phi)$ :

$$
\left(\frac{d W}{d \phi}\right)_{\phi=\phi_{i}}=\left(\frac{d \cos m \theta}{d \sigma}\right)_{\sigma=\sigma_{i}}
$$

where $\phi_{i}<\phi_{i+1}$ and $\sigma_{i}<\sigma_{i+1}$. Together with

$$
\begin{array}{llll}
W\left(\phi_{i}\right) & =0 & & \\
W(\phi) \rightarrow 0 & \text { as } & \phi \rightarrow \phi_{\mp}=\text { finite } \\
\frac{W(\phi)}{\phi} \rightarrow \text { finite } & \text { as } & \phi \rightarrow \phi_{\mp}=\mp \infty
\end{array}
$$

these constraints represent necessary conditions for the equation (20) to have well defined solutions. Given a continuous prepotential function $W(\phi)$ subject to the above constraints the equation (20) is easily integrated in each region $\sigma_{i}<\sigma<\sigma_{i+1}$,

$$
\int \frac{d \phi}{W(\phi)}=\int \frac{d \sigma}{\cos m \theta}+\text { const }
$$

yielding a continuous and monotonous function $\phi(\sigma)$ on the whole $\sigma$-axis. Furthermore, the integration constants in (22) can be chosen to ensure its differentiability. If the function $W(\phi)$ has well defined higher derivatives then $\phi(\sigma)$ will be of the same kind. Therefore, the conditions (21) are also sufficient conditions for the field equations to admit regular m-kink solutions.

Let us consider some examples. The easiest way to construct a potential with given properties is to choose a monotonous function $\phi(\sigma)$, substitute it into (20) to obtain $W(\phi(\sigma))$ as a function of $\sigma$, and then use $\sigma=\sigma(\phi)$ to find $W(\phi)$ itself. Taking the linear dilaton vacuum $\phi=\sigma$ as a starting point we thus find

$$
W(\phi)=W_{m}(\phi) \equiv \cos [m \theta(\phi)]
$$

In particular, the one-kink and two-kink prepotentials are given by

$$
W_{1}(\phi)=1-\frac{2}{1+\phi^{2}} \quad W_{2}(\phi)=1-\frac{8 \phi^{2}}{\left(1+\phi^{2}\right)^{2}}
$$


The potential $V(\phi)$ is determined from (19) up to a multiplicative constant.

As our second example we shall consider the dilaton field of the form $\phi=\theta(\sigma)$. It follows then that

$$
W(\phi)=(1-\cos \phi) \cos m \phi
$$

In its domain $D=(0,2 \pi)$ this function has $2 m$ zeros and consequently admits $m$-kink solutions. Notice, however, that, restricted to the domain between two neighbouring zeros, like, for example, $D_{0} \equiv(\pi / 2 m, 3 \pi / 2 m)$, the prepotential function $W(\phi)$ has no zeros at all and the equation (22) has regular $m=0$ solutions. If we take $D_{1} \equiv(\pi-3 \pi / 2 m, \pi+3 \pi / 2 m)$ as the domain of our function $W(\phi)$ we see that the conditions (21) for the existence of $m=1$ kinks are fulfilled up to a multiplicative constant. Since the potential function $V(\phi)$ $i s$ determined only up to a multiplicative constant, we conclude that the field equations possess one-kink solutions. Thus, the choice (24) of the prepotential $W(\phi)$ ensures the existence of three inequivalent kink configurations.

In the end, let us comment on the possibility to have a potential which would allow for an arbitrary number of inequivalent kink configurations. If $D_{m}(m=0,1, \ldots, k)$ are disjoint finite domains of the prepotential functions $W_{m}(\phi)$ then it is possible to construct the function $W(\phi)$ with the domain $D \supset \bigcup_{m} D_{m}$ such that its restriction to $D_{m}$ is exactly $W_{m}$. If $W_{m}$ admits $m$-kink solutions it follows that $W$ will admit $k+1$ inequivalent kink configurations with kink numbers taking values from 0 to $k$. The theory of this kind will possess $k-1$ types of nonsingular black holes. Our examples (23) and (24) describe nonsingular black holes in different background geometries. The first is asymptotically flat spacetime while the second is a spacetime of constant negative curvature.

\section{Concluding remarks}

We have studied in this paper the properties of two dimensional gravitational kinks as solutions of some simple physical models. In section 2 we have proved that spacetimes of $R^{1} \times S^{1}$ topology admit kink metric configurations of constant curvature. In particular, flat gravitational kinks of arbitrary kink number exist on a cylinder. Viewed as nonsingular black holes, however, these solutions are not appropriate since the horizons are uniformly disposed over the space. To study more realistic black hole solutions we switched our attention in section 3 to spacetimes of trivial topology. We have proved the existence of $m=1$ flat gravitational kinks and argued that these cannot be black hole solutions since it is possible to transform them away. The corresponding coordinate transformations necessarily move the points of spatial infinity. The formal $|m|>1$ kink solutions of the equation $R=0$, valid in regions between the horizons, have been shown to inevitably 
contain singularities. It has been explained in detail why it is impossible to transform $|m|>1$ kinks away. Finally, a simple model of dilaton gravity theory has been considered. It has been shown that a nontrivial potential function is needed if one wants the theory to accommodate nonsingular black hole solutions. The general form of the potential has been found to ensure the existence of stationary gravitational kinks and some examples have been given. We have also proved the possibility of choosing the potential function which admits an arbitrary number of inequivalent kink configurations.

\section{Acknowledgements}

We wish to thank R. Panajotović for her help in making the illustrations. This work has been supported in part by the Serbian Research Foundation, Yugoslavia. 


\section{References}

[1] Finkelstein D and Misner C W 1959 Ann. Phys., NY 6230

[2] Williams J G and Zia R K P 1973 J. Phys. A 61

[3] Finkelstein D and McCollum G 1975 J. Math. Phys. 162250

[4] Whiston G H 1981 J. Phys. A 142861

[5] Clément G 1984 Gen. Rel. Grav. 16 131, 477, 491; 198618137

[6] Harriott T A and Williams J G 1986 J. Math. Phys. 272706

[7] Dunn K A and Williams J G 1989 J. Math. Phys. 3087

[8] Dunn K A 1990 Gen. Rel. Grav. 22507

[9] Finkelstein D 1991 Prehistory of the black hole Georgia Institute of Technology preprint

[10] Finkelstein D 1966 J. Math. Phys. 71218

[11] Dunn K A, Harriott T A and Williams J G 1992 J. Math. Phys. 331437

[12] Trodden M, Mukhanov V F and Brandenberger R H 1993 Phys. Lett. 316B 483

[13] Altshuler B 1990 Class. Quant. Grav. 7189

[14] Morgan D 1991 Phys. Rev. D 433144

[15] Dymnikova J 1992 Gen. Rel. Grav. 24235

[16] Mukhanov V and Brandenberger R 1992 Phys. Rev. Lett. 681969

[17] Banks T and O’Loughlin M 1992 Rutgers preprint RU-92-61

[18] Brandenberger R, Mukhanov V and Sornborger A 1993 Phys. Rev. D 481629

[19] Chan K C K and Mann R B 1995 Class. Quant. Grav. 121609 


\section{Figure captions}

Figure 1. The surface $g=0$ in the space of metric components

Figure 2. One-kink and two-kink light cone configurations

Figure 3. Spacelike and timelike coordinate lines for a one-kink metric

Figure 4. Spacelike and timelike lines of a two-kink metric 
This figure "fig1-1.png" is available in "png" format from: http://arxiv.org/ps/gr-qc/9601018v1 
This figure "fig1-2.png" is available in "png" format from: http://arxiv.org/ps/gr-qc/9601018v1 
This figure "fig1-3.png" is available in "png" format from: http://arxiv.org/ps/gr-qc/9601018v1 
This figure "fig1-4.png" is available in "png" format from: http://arxiv.org/ps/gr-qc/9601018v1 